\title{
Reassessment of Corticospinal Tract Regeneration in Nogo-Deficient Mice
}

\author{
Jae K. Lee, ${ }^{1}$ Andrea F. Chan, ${ }^{1}$ Sen M. Luu, ${ }^{1}$ Yuhong Zhu, ${ }^{1}$ Carole Ho, ${ }^{2}$ Marc Tessier-Lavigne, ${ }^{2}$ and Binhai Zheng ${ }^{1}$ \\ ${ }^{1}$ Department of Neurosciences, University of California San Diego, School of Medicine, La Jolla, California 92093, and ${ }^{2}$ Division of Research, Genentech Inc., \\ South San Francisco, California 94080
}

The myelin-derived neurite growth inhibitor Nogo has been proposed to play a major role in blocking axon regeneration in the CNS after injuries. However, past studies have produced mixed results regarding the regenerative phenotype of various Nogo-deficient mouse lines after experimental spinal cord injury. Two lines did not display enhanced corticospinal tract (CST) regeneration, and one displayed modest regeneration. A fourth line, a Nogo-A,B gene-trap mutant, was instead reported to exhibit extensive CST regeneration, but the results were later found to be inadvertently confounded with an axon labeling artifact. Of the four Nogo mutant lines studied so far, three continue to express some isoform(s) of Nogo, leaving open the question whether any remaining Nogo protein contributes to the modest regenerative phenotype reported in some. The remaining Nogo mutant line studied was confounded by the unexplained rescue of embryonic lethality associated with this mutation. To gain a better understanding of the contribution of Nogo as an inhibitor of regeneration of CNS axons, and particularly CST axons, we reanalyzed the Nogo-A,B gene-trap mutant line and analyzed a novel, fully viable Nogo deletion mutant line that is null for all known isoforms of Nogo. Our analyses failed to reveal any enhanced CST regeneration after experimental spinal cord injury in either line. These results indicate that Nogo alone does not account for lack of CST regeneration and have implications for current therapeutic development for spinal cord injury in humans by targeting Nogo.

\section{Introduction}

The limited ability of CNS axons to regenerate after injury has been partially attributed to inhibitors of axon regeneration in CNS myelin (Yiu and He, 2006). Of the three extensively characterized myelin-derived axon growth inhibitors [Nogo, myelinassociated glycoprotein (MAG), and oligodendrocyte myelin glycoprotein (OMgp)], Nogo has received the most attention as a therapeutic target for spinal cord injury (Gonzenbach and Schwab, 2008). Treatment with antibody IN-1 has been reported to promote corticospinal tract (CST) regeneration in rodents (Schnell and Schwab, 1990; Brösamle et al., 2000), but this antibody is not specific to Nogo (Caroni and Schwab, 1988a,b). More selective antibodies promote more limited regeneration in rats (Liebscher et al., 2005), and the statistical significance of their reported effect on regeneration in primates (Freund et al., 2006) has been questioned (Ho and Tessier-Lavigne, 2006). A peptide that disrupts the interaction between Nogo and Nogo receptor $(\mathrm{NgR} 1)$ promoted CST regeneration in some experiments

\footnotetext{
Received April 20, 2009; revised May 26, 2009; accepted June 8, 2009.

This work is supported by research grants from the Roman Reed Spinal Cord Injury Research Fund of California and National Institutes of Health-National Institute of Neurological Disorders and Stroke Grant R01NS054734. J.K.L. is supported by Ruth L. Kirschstein National Research Service Award Postdoctoral Fellowship F32NS056697. We thank Oswald Steward for providing the Nogo-A,B gene-trap mutant and Han Lin, Nona Velarde, and Helen Rayburn for technical assistance.

Correspondence should be addressed to Dr. Binhai Zheng, Department of Neurosciences, University of California San Diego, 9500 Gilman Drive, MC 0691, La Jolla, CA 92093-0691. E-mail: binhai@ucsd.edu.

D0I:10.1523/JNEUROSCI.1864-09.2009

Copyright $\odot 2009$ Society for Neuroscience $\quad$ 0270-6474/09/298649-06\$15.00/0
}

(GrandPré et al., 2002; Li and Strittmatter, 2003) but not in others (Steward et al., 2008).

Genetic studies in mice have been used to interrogate Nogo function more decisively but have produced mixed results (Zheng et al., 2006). Three groups collectively reported a broad, puzzling spectrum of CST regeneration phenotypes in four Nogo mutant lines. A first group did not observe any enhanced CST regeneration in a Nogo-A,B targeted mutant or a C-terminal targeted mutant that is null for Nogo-C and severely hypomorphic for Nogo-A,B (Zheng et al., 2003). However, all viable C-terminal mutants analyzed had been derived from a single, rare escaper mutant mouse that spontaneously arose in an otherwise homozygous lethal line. It is not known how the reversal of the lethality occurred and whether it was associated with any confounding compensatory mechanism pertinent to axon regeneration.

The second group reported a trend for enhanced CST regeneration beyond the lesion site in a Nogo-A targeted mutant, but this trend did not reach statistical significance (Simonen et al., 2003). The lack of robust regeneration in this mutant has been attributed to two confounding factors: the mixed genetic background originally analyzed (Dimou et al., 2006) and the marked, unintended upregulation of Nogo-B in this mutant (Simonen et al., 2003).

The third group initially reported massive CST regeneration in a Nogo-A,B gene-trap mutant as evidenced by the presence of extensive ectopic fibers in the lateral and ventral white matter both above and below the level of injury (Kim et al., 2003). However, this evidence was later explained by a labeling artifact that 
occurs when the tracer is injected too deep into the brain and leaks into the ventricles (Steward et al., 2007).

To minimize confounding factors in previous Nogo mutant studies and to clarify the role of Nogo in CST regeneration, we reanalyzed the Nogo-A,B gene-trap mutant in the absence of artifactual labeling, and we analyzed a novel, fully viable Nogo deletion mutant that lacks all known isoforms of Nogo. We found that neither the Nogo-A,B gene-trap mutant nor the Nogo deletion mutant exhibits enhanced CST regeneration after spinal cord injury.

\section{Materials and Methods}

Both the Nogo-A,B gene-trap mutation (Kim et al., 2003) and the Nogo deletion mutation (this study; for details, see Results) were made in mouse embryonic stem cells of the 129/ SvEvBrd genetic background. The Nogo-A,B gene-trap mutation has been backcrossed to C57BL/6 three to six times (Kim et al., 2003). The Nogo deletion mutation was bred to an Actin-Cre deleter line of the FVB background (Lewandoski et al., 1997) and backcrossed to C57BL/6 three times. All experimental procedures, including surgery, behavioral observation, and axon quantification, were done in a blind manner. Female mice ( $8-10$ weeks) were used for surgery. The surgical procedure were as described previously (Zheng et al., 2003) with minor modifications (supplemental data, available at www.jneurosci.org as supplemental material). All procedures were approved by the Institutional Animals Care and Use Committee at University of California, San Diego.

Antibodies used for Western blot analysis include the following: rabbit anti-Nogo-A at 1:500 (Zheng et al., 2003); goat anti-NgR1 (R \& D Systems); goat anti-MAG at 1:1000 (R \& D Systems); rat anti-OMgp at $1: 1000$ (R \& D Systems); and mouse anti- $\alpha$-tubulin at 1:1000 (Sigma). Histology and quantification were performed as described previously (Zheng et al., 2003) with minor modifications (supplemental data, available at www.jneurosci.org as supplemental material).

\section{Results}

\section{Analysis of Nogo-A,B gene-trap mutant mice}

We subjected Nogo-A,B gene-trap mutants (Kim et al., 2003) together with heterozygous littermates to a T8 dorsal hemisection model of spinal cord injury and traced the CST unilaterally with biotinylated dextran amine (BDA) as described previously (Zheng et al., 2003) (supplemental data, available at www. jneurosci.org as supplemental material). To avoid the labeling artifacts described previously (Steward et al., 2007), we injected the BDA tracer with care, so as not to accidentally penetrate the cortex with the injection needle, with one important difference: in the current study, we lowered the injection needle directly 0.6 $\mathrm{mm}$ into the cortex, whereas in the previous study (Steward et al., 2007), the needle was first lowered below $0.5 \mathrm{~mm}$ and then withdrawn back to the final position at $0.5 \mathrm{~mm}$. Although the previous protocol gave rise to labeling artifacts in a subset of animals, as evidenced by ectopic labeled fibers with a hollow appearance in the lateral and ventral white matter above and below the level of injury (Steward et al., 2007), our current protocol avoided the artifactual labeling. This is evidenced by the lack of "ectopic," hollow axons on transverse sections $4 \mathrm{~mm}$ above and $4 \mathrm{~mm}$ below the injury site (supplemental Fig. 1, available at www.jneurosci. org as supplemental material). In contrast, the pattern of artifactual, ectopic fibers can be reproducibly obtained by injecting the tracer deliberately deep into the brain (supplemental Fig. 2, available at www.jneurosci.org as supplemental material). These artifactually labeled axons tend to appear much thicker than the genuinely labeled CST axons (supplemental Fig. 2, available at www.jneurosci.org as supplemental material; compare $B, E$ with $C, D)$. Thus, we concluded that our experimental protocol reliably detects CST axons without any detectable level of labeling artifact. We chose a 3-week survival time to allow for a direct comparison with the published reports in which CST regeneration was detected (Kim et al., 2003; Cafferty et al., 2007).

In both Nogo-A,B gene-trap mutants and heterozygous control mice, the main CST tract containing numerous retraction bulbs approached the injury site with a few axons extending closer but rarely beyond the injury site (Fig. $1 A, B$ ). This is typical of the behavior of CST axons after a dorsal hemisection injury in mice. We used a quantification scheme on sagittal sections described previously (Simonen et al., 2003; Zheng et al., 2003). Briefly, labeled CST axons at different distances rostral and caudal to the injury on the sagittal sections were counted and averaged over multiple sections around the main CST; these numbers were then normalized against the total axons labeled in the medulla. This analysis did not reveal any significant differences between Nogo-A,B gene-trap mutants and heterozygous littermates in areas caudal as well as rostral to the injury site (Fig. 1C). Thus, under conditions in which artifactual labeling is avoided, enhanced CST regeneration is not readily detected in the Nogo-A,B gene-trap mutant in a dorsal hemisection model of spinal cord injury.

Throughout the testing period, both Nogo-A,B gene-trap mutants and heterozygous control mice displayed a similar degree of locomotor recovery as measured by a modified Basso-Beattie- 
A

Wild type

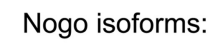

A, B A C

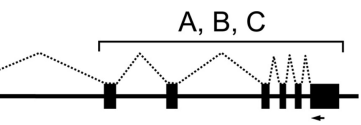

\section{Targeting C-terminus}

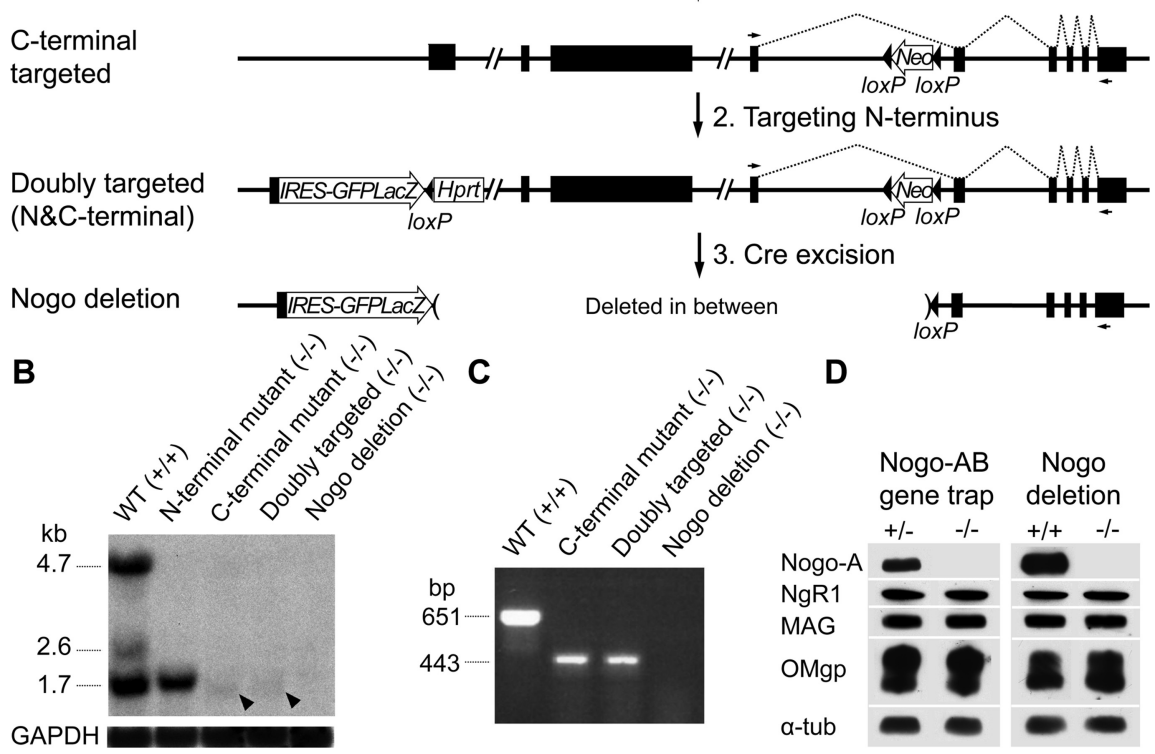

Figure 2. Generation and molecular characterization of the Nogo deletion mutant. $A$, The strategy to obtain the Nogo deletion allele via two consecutive gene targeting steps followed by Cre-mediated excision. A, B, C above the exons (black bars) refer to Nogo isoforms. Dotted lines, Splicing pattern for the Nogo-C transcript; Neo, neomycin resistance gene; Hprt, hypoxanthine phosphoribosyltransferase gene; IRES, internal ribosomal entry site; GFPLacZ, green fluorescent protein and $\beta$-galactosidase fusion gene; small arrows, primers used to analyze the Nogo-C transcript by RT-PCR. Two Frt sites flanking Hprt are omitted for simplicity. B, Northern blot with a C-terminal common probe. Nogo-A,B, C transcripts run at 4.7, 2.6, and $1.7 \mathrm{~kb}$, respectively. Arrowheads indicate a slightly shorter, mutated form of Nogo-C transcript. WT, Wild type. C, RT-PCR to confirm the absence of Nogo-C transcript in the Nogo deletion mutant. $\boldsymbol{D}$, Western blot analysis on Nogo-A, NgR1, MAG, and OMgp in Nogo-A, B gene-trap and Nogo deletion mutants. $\alpha$-tub, $\alpha$-Tubulin.

Bresnahan (BBB) locomotor score (Basso et al., 1995; Zheng et al., 2003). One day after injury, mice of both genotypes displayed, on average, slight movement of all three joints, which improved to consistent plantar stepping with frequent coordination of the forelimbs and hindlimbs by $7 \mathrm{~d}$ after injury (Fig. $1 D$ ). This level of locomotor recovery was maintained until the end of the experiment, comparable with what was observed previously in this injury model (Zheng et al., 2003).

\section{Generation and analysis of Nogo deletion mutant mice}

All previous Nogo mutant studies were confounded by either an incomplete knock-out of all isoforms or an unexplained rescue of embryonic lethality associated with the mutation. In particular, the presence of Nogo-C in the Nogo-A,B targeted mutant (Zheng et al., 2003), the Nogo-A,B gene-trap mutant (Kim et al., 2003), and the Nogo-A targeted mutant (Simonen et al., 2003) could explain a lack of more extensive CST regeneration in these mutants. The only Nogo mutant analyzed in which all three main isoforms (Nogo-A,B,C) are disrupted (either null or hypomorph), the Nogo C-terminal mutant, was derived from a rare survivor of an otherwise lethal line (Zheng et al., 2003). This raised the question whether any compensatory mechanisms that were responsible for the rescue of the lethality in this mutant may have also contributed to masking a regenerative phenotype. To address these concerns, we generated and analyzed a Nogo deletion mutant that lacks all known Nogo isoforms (including Nogo-A,B,C), is fully viable, and is therefore not complicated by any associated embryonic lethality.
Alternative promoter usage and the size of the Nogo gene made it impossible to generate a clean Nogo deletion allele with a single round of conventional gene targeting. Using two targeting vectors that we used previously to generate the Nogo C-terminal mutant and the Nogo-A,B targeted mutant (i.e., the Nogo N-terminal mutant), respectively (Zheng et al., 2003), we generated an N-terminal and C-terminal double-targeted mutant by consecutive targeting in the same mouse embryonic stem cell line (Fig. 2). The double-targeted allele was transmitted through the germ line and bred to a Cre deleter mouse (Lewandoski et al., 1997) to obtain the Nogo deletion allele (Fig. 2A). When bred to homozygosity, the Nogo deletion mutants were viable and obtained in a Mendelian ratio (10 wild-type, 13 heterozygous, and 9 homozygous mutants) from intercrosses between heterozygous mice, demonstrating that Nogo is not required for viability. The Nogo deletion mutants were fertile and morphologically indistinguishable from their wild-type or heterozygous littermates.

To confirm that the Nogo deletion mutant is null for Nogo-A,B,C, we performed Northern blot, reverse transcription (RT)PCR, and Western blot analyses on adult brain samples. As expected, Northern blot analysis with a common C-terminal probe detected all three major Nogo transcripts in the wild-type controls, only the Nogo-C transcript in the N-terminal mutant, a residual amount of a slightly shorter form of Nogo-C transcript in the C-terminal mutant and the double-targeted mutant, and no detectable Nogo transcript of any size in the Nogo deletion mutant (Fig. $2 \mathrm{~B}$ ). This result was confirmed with RT-PCR using a Nogo-C-specific primer pair. As expected, we detected a product of normal size in the wild-type control, one of reduced size in the C-terminal targeted mutant and the double-targeted mutant, and no product in the Nogo deletion mutant (Fig. 2C). In addition, a highly specific Nogo-A antibody (Zheng et al., 2003) did not detect any Nogo-A protein in the Nogo deletion mutant (Fig. 2D). These results indicate that the Nogo deletion mutant is null for all known Nogo isoforms. Similar to the Nogo-A,B gene-trap mutant, the expression of other components implicated in the myelin inhibition pathway, such as NgR1, MAG, and OMgp, was not altered in the Nogo deletion mutant (Fig. 2D).

After a dorsal hemisection, CST axons in both the Nogo deletion mutants and age and genetic background-matched wildtype controls displayed typical retraction bulbs just rostral to the injury site with a few axons that approached but rarely passed the injury site (Fig. $3 A, B$ ). Interestingly, quantification of CST axons in the rostral region showed significantly fewer CST axons present in the Nogo deletion mice compared with wild-type controls. A similar trend has also been observed in two previous Nogo mutant studies (Simonen et al., 2003; Zheng et al., 2003). It is not known whether this trend represents a true decrease in rostral sprouting in Nogo mutants or simply reflects the high variability of axonal counts rostral to the injury site. Nevertheless, 

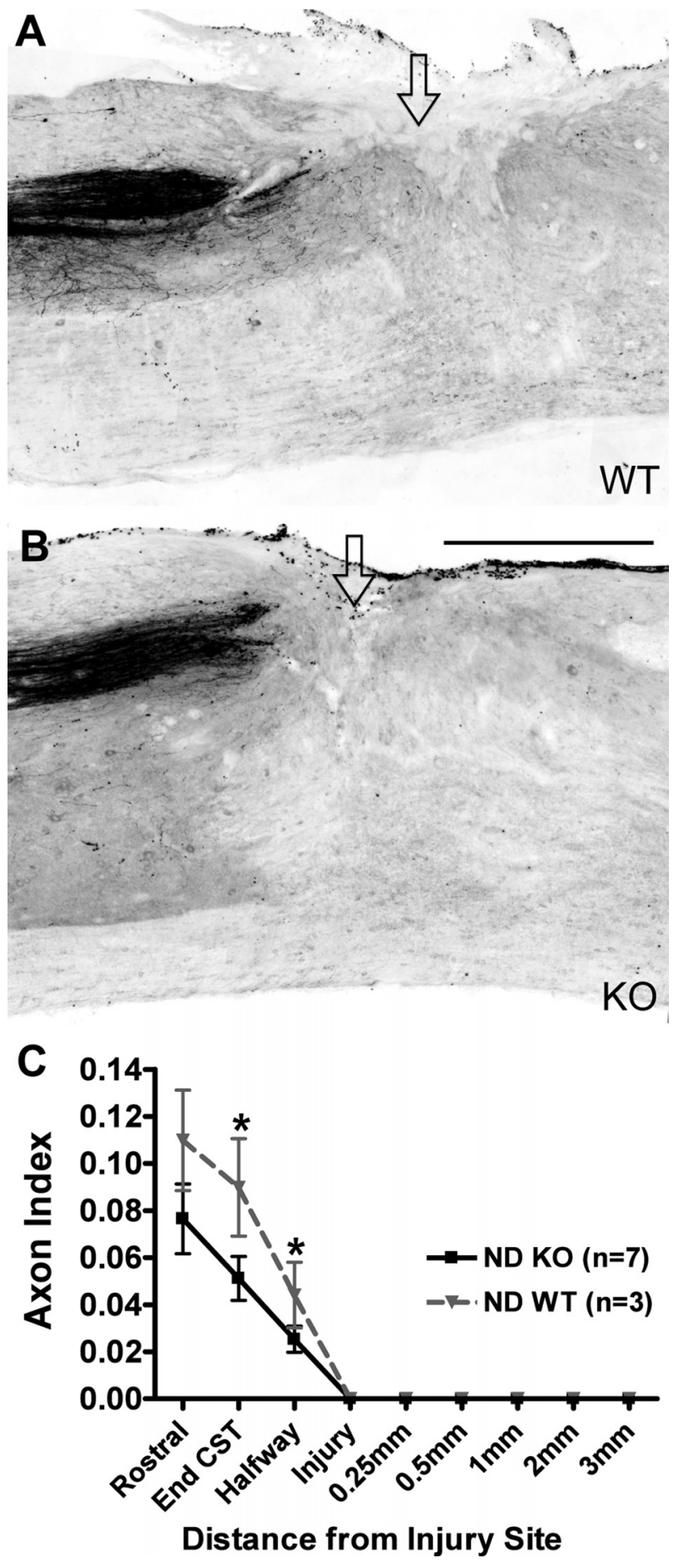

Figure 3. Analysis of Nogo deletion mutant mice for CST regeneration. $\boldsymbol{A}, \boldsymbol{B}$, Representative sagittal spinal cord sections showing the labeled main CST tract approaching but not passing through the injury site (arrow). ND, Nogo deletion; WT, wild type; K0, knock-out. Rostral is to the left. Scale bar, $500 \mu \mathrm{m}$. C, Quantification of CST axons caudal to the injury site did not indicate any significant differences between the two genotypes, but wild-type mice showed significantly more axons at two distance points rostral to the injury site. ${ }^{*} p<0.05$ two-way repeated measures ANOVA with Bonferroni's post hoc test.

there is no significant difference at or below the level of injury between the two genotypes. Serial sagittal sections of the injury site rarely showed any CST axons caudal to the injury site in either the Nogo deletion mutant or wild-type mice. This was also apparent in transverse sections of the caudal spinal cord, which was devoid of any CST axons in both the white and gray matter (supplemental Fig. 1, available at www.jneurosci.org as supplemental material).

\section{Discussion}

Inconsistencies in the results from three initial studies on CST regeneration in various Nogo mutant lines (Kim et al., 2003; Simonen et al., 2003; Zheng et al., 2003) raised intriguing questions of how loss-of-function mutations in the same gene could lead to such varying phenotypes and whether Nogo indeed plays a major role in inhibiting CNS axon regeneration (Woolf, 2003). Variabilities in experimental techniques, the nature of the mutation(s) studied, and the genetic background analyzed were considered as potential explanations for the discrepancies. Previous attempts to address these discrepancies have implicated a genetic background effect (Dimou et al., 2006) and an unexpected axon labeling artifact (Steward et al., 2007). To clarify the role of Nogo in CST regeneration, we asked whether the Nogo-A,B gene-trap mutant line displays enhanced CST regeneration under conditions that avoid artifactual labeling. In addition, we analyzed a novel, fully viable Nogo deletion mutant that lacks all known Nogo isoforms to eliminate the confounding factors of all previous Nogo mutants analyzed. Our results indicate that neither Nogo mutant line exhibits significantly enhanced CST regeneration beyond the injury site in a well established dorsal hemisection model of spinal cord injury.

The presence of extensive ectopic fibers in the lateral and ventral white matter above and below the level of injury was a hallmark phenotype in the initial study supporting robust CST regeneration in the Nogo-A,B gene-trap mutant (Kim et al., 2003). The term ectopic refers to the fact that they were observed at locations unusual for CST axons. The presence of traced axons that take an unusual course or are present at unusual locations constitutes one criterion for regenerated axons (Steward et al., 2003). In mice, a small number of CST axons $(\sim 1-2 \%)$ course down the spinal cord in the dorsolateral white matter (Steward et al., 2004), and occasionally one may detect an even smaller number of CST axons in the ventral white matter along the ventral median fissure (our unpublished observation). However, the ectopic fibers observed in the Nogo-A,B gene-trap mutant were far greater in number (up to $>1000$ axons, which is comparable with the total number of the main CST axons labeled) and formed a pattern that is uncharacteristic for CST axons (Kim et al., 2003).

In fact, this phenotype of ectopic fibers presented the most striking contrast when compared with the lack of enhanced CST regeneration observed in the Nogo-A,B targeted mutant line (Zheng et al., 2003). Differences in lesion severity, mutations, and genetic background were considered as potential causes for the divergent results. Genetic background was unlikely to underlie the striking phenotypic difference, given that both mutants were made with mouse embryonic stem cells of the 129/SvEvBrd strain origin and had been backcrossed to C57BL/6 a few times. Lesion severity or any other variables in spinal surgery were excluded because the lack of regeneration phenotype in the Nogo-A,B targeted mutant was reproduced by the group that initially observed extensive CST regeneration in the Nogo-A,B gene-trap mutant (Kim et al., 2003; Zheng et al., 2003). In terms of the nature of the mutations, both mutant lines lack Nogo-A,B but retain Nogo-C expression. Thus, although the two mutations were made with different approaches (gene trapping vs gene targeting), they appear to have the same effect on the Nogo proteins. If there is any difference, the Nogo-A,B targeted mutant is null for Nogo-A,B 
(Zheng et al., 2003), whereas the Nogo-A,B gene-trap mutant may still express a residual amount of a peptide consisting of the first 309 amino acid residues of Nogo-A, albeit at too low a level to be detected by Western blot, although the transcript encoding this peptide is readily detected by Northern blot (Kim et al., 2003).

A recent study raised a methodological concern regarding the original Nogo-A,B gene-trap mutant study by showing that such a pattern of ectopic fibers can be recapitulated in mice in which BDA is inadvertently injected too deep into the brain and consequently leaks into the ventricles below the cortex (Steward et al., 2007). The tracer then presumably travels to the injury site via CSF and is taken up by the damaged or compromised axons at the injury site, leading to artifactual labeling of non-CST axons. This phenomenon is independent of the genotype, i.e., either a gene-trap mutant or a heterozygous control mouse can exhibit this phenomenon. Even wildtype mice can exhibit the same pattern of ectopic fibers when the tracer is deliberately injected deep into the brain (Steward et al., 2007). In fact, we confirmed that this labeling artifact is rather reproducible in our hands (supplemental Fig. 2, available at www.jneurosci.org as supplemental material). However, neither the study describing the labeling artifact (Steward et al., 2007) nor a reanalysis of the data from the initial Nogo-A,B gene-trap mutant study (Cafferty et al., 2007) addressed whether this mutant displays enhanced CST regeneration under conditions that avoid artifactual axon labeling.

To address this confounding factor, we reanalyzed the genetrap mutant while being attentive to the BDA methodological concerns. By carefully positioning the injection needle, we practically avoided any labeling artifacts (i.e., the hollow, ectopic fibers in the lateral and ventral white matter), even when the injection is performed immediately after spinal cord injury. Our data failed to detect any significant increase in CST axons caudal to the injury site in the mutants. In addition, we did not observe any enhancement in behavioral recovery in the mutants as assessed by an open-field locomotion test. Thus, we were not able to reproduce the results previously reported in these mice and conclude that the Nogo-A,B gene-trap mutant does not exhibit enhanced CST regeneration.

Another confounding factor in previous Nogo mutant studies is that all previous mutants studied either still express one or more Nogo isoforms or is complicated by the unexplained rescue of lethality caused by the mutation, which could be associated with compensatory mechanisms pertinent to axon regeneration. We therefore generated a Nogo deletion mutant line via Cre-loxP-mediated recombination after two consecutive steps of gene targeting. This Nogo deletion line is confirmed to be a complete Nogo null allele, lacking the expression of all known Nogo isoforms. It is fully viable, thus not complicated by any associated embryonic lethality. Nevertheless, even this line does not exhibit detectable enhancement of CST regeneration after spinal cord injury. Although this report focuses on axon regeneration, in an open-field study, we did not detect improved locomotion in this mutant compared with controls (data not shown).

In summary, our data indicate that deleting Nogo isoforms, even all isoforms together, does not elicit enhanced CST regeneration beyond the lesion site in mice. Thus, it appears that genetic studies in Nogo mutant mice collectively fail to recapitulate the positive effects reported with anti-Nogo antibodies (Liebscher et al., 2005; Freund et al., 2006). Whether the difference between genetic knock-out experiments and anti-
Nogo antibody treatment is attributable to chronic versus acute disruption of gene function remains unknown. Alternatively, Nogo antibody treatment may not promote functional recovery or axon regeneration simply by neutralizing Nogo; for instance, antibodies might recruit immune effector cells. With the caveat that germ-line mutations may cause developmental compensation (although we did not find evidence for this so far based on our limited assays), we conclude that Nogo alone cannot account for the poor CST regeneration after injury. It should be noted that our analysis focused on axon regeneration rather than sprouting or plasticity that may also contribute to behavioral recovery (Cafferty et al., 2008). Nevertheless, the data presented here illustrate our still limited understanding of the contribution of a major myelin-derived axon growth inhibitor in a well defined experimental paradigm and has important implications for ongoing clinical trials using Nogo-A antibodies to treat spinal cord injury in humans.

\section{References}

Basso DM, Beattie MS, Bresnahan JC (1995) A sensitive and reliable locomotor rating scale for open field testing in rats. J Neurotrauma 12:1-21.

Brösamle C, Huber AB, Fiedler M, Skerra A, Schwab ME (2000) Regeneration of lesioned corticospinal tract fibers in the adult rat induced by a recombinant, humanized IN-1 antibody fragment. J Neurosci 20:8061-8068.

Cafferty WB, Kim JE, Lee JK, Strittmatter SM (2007) Response to correspondence: Kim et al., "axon regeneration in young adult mice lacking Nogo-A/B.” Neuron 38, 187-199. Neuron 54:195-199.

Cafferty WB, McGee AW, Strittmatter SM (2008) Axonal growth therapeutics: regeneration or sprouting or plasticity? Trends Neurosci 31:215-220.

Caroni P, Schwab ME (1988a) Two membrane protein fractions from rat central myelin with inhibitory properties for neurite growth and fibroblast spreading. J Cell Biol 106:1281-1288.

Caroni P, Schwab ME (1988b) Antibody against myelin-associated inhibitor of neurite growth neutralizes nonpermissive substrate properties of CNS white matter. Neuron 1:85-96.

Dimou L, Schnell L, Montani L, Duncan C, Simonen M, Schneider R, Liebscher T, Gullo M, Schwab ME (2006) Nogo-A-deficient mice reveal strain-dependent differences in axonal regeneration. J Neurosci 26:5591-5603.

Freund P, Schmidlin E, Wannier T, Bloch J, Mir A, Schwab ME, Rouiller EM (2006) Nogo-A-specific antibody treatment enhances sprouting and functional recovery after cervical lesion in adult primates. Nat Med 12:790-792.

Gonzenbach RR, Schwab ME (2008) Disinhibition of neurite growth to repair the injured adult CNS: focusing on Nogo. Cell Mol Life Sci 65:161-176.

GrandPré T, Li S, Strittmatter SM (2002) Nogo-66 receptor antagonist peptide promotes axonal regeneration. Nature 417:547-551.

Ho C, Tessier-Lavigne M (2006) Challenges to the report of Nogo antibody effects in primates. Nat Med 12:1232; author reply 1232-1233.

Kim JE, Li S, GrandPré T, Qiu D, Strittmatter SM (2003) Axon regeneration in young adult mice lacking Nogo-A/B. Neuron 38:187-199.

Lewandoski M, Meyers EN, Martin GR (1997) Analysis of Fgf8 gene function in vertebrate development. Cold Spring Harb Symp Quant Biol 62:159-168.

Li S, Strittmatter SM (2003) Delayed systemic Nogo-66 receptor antagonist promotes recovery from spinal cord injury. J Neurosci 23:4219-4227.

Liebscher T, Schnell L, Schnell D, Scholl J, Schneider R, Gullo M, Fouad K, Mir A, Rausch M, Kindler D, Hamers FP, Schwab ME (2005) Nogo-A antibody improves regeneration and locomotion of spinal cord-injured rats. Ann Neurol 58:706-719.

Schnell L, Schwab ME (1990) Axonal regeneration in the rat spinal cord produced by an antibody against myelin-associated neurite growth inhibitors. Nature 343:269-272.

Simonen M, Pedersen V, Weinmann O, Schnell L, Buss A, Ledermann B, Christ F, Sansig G, van der Putten H, Schwab ME (2003) Systemic deletion of the myelin-associated outgrowth inhibitor Nogo-A improves re- 
generative and plastic responses after spinal cord injury. Neuron 38:201-211.

Steward O, Zheng B, Tessier-Lavigne M (2003) False resurrections: distinguishing regenerated from spared axons in the injured central nervous system. J Comp Neurol 459:1-8.

Steward O, Zheng B, Ho C, Anderson K, Tessier-Lavigne M (2004) The dorsolateral corticospinal tract in mice: an alternative route for corticospinal input to caudal segments following dorsal column lesions. J Comp Neurol 472:463-477.

Steward O, Zheng B, Banos K, Yee KM (2007) Response to: Kim et al., "axon regeneration in young adult mice lacking Nogo-A/B." Neuron 38, 187199. Neuron 54:191-195.
Steward O, Sharp K, Yee KM, Hofstadter M (2008) A re-assessment of the effects of a Nogo-66 receptor antagonist on regenerative growth of axons and locomotor recovery after spinal cord injury in mice. Exp Neurol 209:446-468.

Woolf CJ (2003) No Nogo: now where to go? Neuron 38:153-156.

Yiu G, He Z (2006) Glial inhibition of CNS axon regeneration. Nat Rev Neurosci 7:617-627.

Zheng B, Ho C, Li S, Keirstead H, Steward O, Tessier-Lavigne M (2003) Lack of enhanced spinal regeneration in Nogo-deficient mice. Neuron 38:213-224.

Zheng B, Lee JK, Xie F (2006) Genetic mouse models for studying inhibitors of spinal axon regeneration. Trends Neurosci 29:640-646. 